\title{
ELEMENTOS DE LA PRAXIS Y DEL CORPUS DEL CONOCIMIENTO ETNOECOLÓGICO TSEltal en COMUnidades de la Sierra Norte de Chiapas
}

\author{
Elements of the Praxis and Corpus of Tseltal Ethnological Knowledge in Communities of the Northern \\ Sierra of Chiapas
}

José Ramón Rodríguez-Moreno

Resumen: Durante décadas, las estrategias de uso y manejo de los ecosistemas, como las que practican los tseltales en la Sierra Norte de Chiapas y otros grupos étnicos en el mundo, han sido insistentemente descritas por una visión occidental "científica" como sistemas de baja productividad y derrochadoras de recursos naturales. En este estudio se reseñan algunos aspectos del cosmos, corpus y praxis del conocimiento que se posee en comunidades tseltales, a partir del hilo conductor de un rubro específico del campo mexicano, el maíz y de otras manifestaciones del conocimiento local, que permiten desmontar la marginación del sistema de conocimiento local con que lo ha revestido la visión occidental.

Palabras clave: etnoecología tseltal, conocimiento local, variedades de maíz.

Abstract: During the last century, the farming productive strategies implemented by ethnics communities around the world, conjunctively with the way they use their natural resources, has been stigmatized by a western "scientific" vision, as wasteful and harmful to the cosystems. This is also the case for the tseltal communities of the Northern Sierra of Chiapas. The research reviews and surfaces some aspects of the cosmos, corpus and praxis of the tseltal local ecological knowledge, using as reference the milpa production system and other manifestations of this "local and ethnic knowledge" to dismount this "rational" perspective of western reductionism.

Keywords: tseltal ethnoecology, local knowledge, maize varieties.

José Ramón Rodríguez Moreno, doctor en antropología social por la Universidad Iberoamericana, México. Actualmente, realiza un posdoctorado en CIESAS-Sureste, México. con convenio CONACyT. Temas de especialización: soberanía alimentaria y sistemas locales de subsistencia.Correo electrónico: bakelman@gmail.com.

Enviado a dictamen: 18 de diciembre de 2012.

Aprobación: 09 de julio de 2013

Revisiones: 2. 


\section{Introducción}

$\amalg$ s un rasgo característico de la supuesta unidad del mundo moderno considerar los modos de producción agraria de los grupos indígenas como portadores de un conocimiento insignificante, poco coherente e incomprensible, necesariamente destinado a ser sustituido por estrategias mejoradas de tecnología y ciencia occidental. Se erige con esto la "racionalidad científica como el criterio de demarcación entre lo que es válido como conocimiento y lo que no es" (De Souza, 2007: 208) y, en tal sentido, todo lo ajeno a esta episteme es desperdiciable e inútil para ayudar a desmontar las condiciones de subsistencia crítica y la marginalidad social en las cuales estos grupos humanos han estado sumidos.

Una valoración sobre este tema la realiza Stephen Marglin (1991, citado por Richards, 1993), quien ha llamado la atención sobre las peculiaridades históricas asociadas a la estricta segregación entre episteme y techne como formas de conocimiento del mundo occidental, así como sobre quienes practican la una o la otra. Reporta Richards (1993) cómo, por ejemplo, en la sociedad inglesa, cuna de la Revolución Industrial, desde el siglo XIX insistían en monopolizar y separar el pensamiento reflexivo, intelectual y científico, es decir, "superior", del conocimiento práctico y menospreciado que ejercían y practicaban los constructores, carpinteros, alfareros o torneros, es decir, los artesanos. De la misma manera, salvando las distancias y los tiempos, ésta es la racionalidad que opera en la ciencia occidental y en el conocimiento étnico, una perspectiva que ha "generado conocimiento e ignorancia sistemática respecto a cómo los grupos humanos rurales asociados al agro actúan dentro del mundo moderno" (Ploeg, 2010: 39).

Durante décadas, las estrategias de uso y manejo de ecosistemas como las que practican los tseltales en la Sierra Norte de Chiapas y otros grupos étnicos de distintas regiones del mundo, han sido insistentemente descritas por esa misma visión occidental científica como sistemas de baja productividad, "derrochadoras de recursos naturales y en consecuencia, los que las practican como destructores de los ecosistemas naturales" (Toledo et al., 2003). En apariencia, esta visión parcializada fue consecuencia de la aplicación de estereotipos occidentales a la interpretación de las prácticas utilizadas en el trópico húmedo. Desde esta perspectiva, se ignoraban las prácticas autóctonas y el manejo de procesos ecológicos desarrollados localmente, ya que sólo se reconocía la aproximación centrada en el cientificismo de lo agroeconómico.

El presente ensayo tiene como finalidad reseñar algunos aspectos del corpus y de la praxis del conocimiento que se posee en comunidades tseltales de la Sierra Norte de Chiapas (ver mapa 1) a partir del eje fundamental de la subsistencia en el campo mexicano, la milpa y el maíz, dentro de otras manifestaciones del conocimiento local o art de la localité (Ploeg, 1990), que claramente permiten develar cómo la visión occidental ha marginado este tipo de conocimiento. Se intenta mostrar cómo las visiones epistémicas y los significados que practica "esta clase incómoda" (Ploeg, 2010: 40), manifiestos en distintas partes del mundo, se encuentran en comunidades tseltales, que validan y refuerzan sus hallazgos y argumentos por encima de la artificialidad e impostura occidental sobre lo que es el conocimiento científico y quién realmente lo practica.

Se examinarán en primer lugar algunos elementos generales del conocimiento local tseltal, "conocimiento total y autoconocimiento" (Gandarilla, 2011: 207), a partir de dos evaluaciones etnográficas desarrolladas en dos comunidades tseltales de los municipios de Chilón y Sitalá en la Sierra Norte de Chiapas: Sanulha' y San Agustín el Mirador. Adicionalmente, se destacarán varias especificidades sobre prácticas de cultivo del maíz, así como sobre el uso de distintas variedades, la selección de semillas, las estrategias ecológicas de secuencialidad en el uso del suelo, la caracterización edafológica y las taxonomías locales, así como las asociaciones semillas-suelos que ponen en práctica los productores tseltales.

Por último, se prestará atención a la presencia en la zona de algunas instituciones cívico-religiosas de fomento al tejido y densificación social que giran en torno a las actividades de la misión jesuita de Bachajón, como los jcanan lumk'inal o cuidadores de la madre tierra, 
cargo incorporado al tradicional sistema de cargos tseltal que opera en la región de estudio, que enfatiza prácticas productivas agroecológicas, y que impulsa con ello contextos de desarrollo a partir de lo que De Souza llama "ecología de saberes", es decir, "la práctica de agregación de la diversidad a través de la promoción de interacciones sustentables entre entidades parciales y heterogéneas" (De Souza, 2007: 182).

\section{Revalorización de la praxis, corpus y cosmos como sabiduría local. Las llaves significantes de un conocimiento total}

Como se ha reportado ampliamente en estudios mesoamericanos, "la cosmovisión de los pueblos indígenas concibe al universo, a la naturaleza, a la sociedad y al cuerpo humano, como semejante, como emparentado y como relacionado en una reciprocidad equilibrada" (Barabas, 2003: 24). En este espacio de diversidad cultural, prácticamente cada comunidad y cada región étnica implementan esquemas particulares de ritualidad cosmogónica y de prácticas rutinarias de existencia, "espacios de cotidianidad que abarcan todos los ámbitos de la producción humana" (Broda et al., 2007: 321). Un sistema de vida en donde los esquemas indisolubles praxis, corpus y cosmos llenan el paisaje de complejidady "definen los espacios culturales, mediante procesos continuos de reelaboración simbólica" (Broda et al., 2007: 322), dándoles un carácter de organicidad y unicidad, en donde se hace banal y poco pertinente implementar las lógicas paradigmáticas de occidente, con sus "polaridades binarias sociedad-naturaleza" (De Souza, 2009: 207).

En estos contextos, se estructura un tipo de conocimiento que se produce como "conocimiento total" (Gandarilla, 2011: 207), donde el vínculo entre la cosmovisión, el ritual y la observación de la naturaleza, condicionados por el medioambiente, genera un mosaico de saberes o "ecología de saberes" (De Souza, 2009: 207) en el cual la práctica de agregación de la diversidad, a través de interacciones de distintos naturaleza y origen, ha confrontado, desde la llegada de los europeos, la monocultura de la ciencia moderna con el complejo simbolismo de estos lugares de "geografías sagradas" (Broda y Báez, 2001: 16).

Plantea Cardoso de Oliveira (2007) que es difícil llegar a conocer, a partir de las rutas científicas del análisis occidental, "las categorías fundamentales de entendimiento de estas culturas" (Cardoso de Oliveira, 2007: 17). Una razón es la ausencia de abstracciones en sus prácticas concretas y en su verbalización, racionalidad operativa con que normalmente procede el método científico. Esta falta de "llaves de significados" (Ingold, 2000: 22) es consecuencia de la manera como Occidente discrimina el universo entre "forma y substancia" (Bateson, 1973: 122). Así pues, para Bateson (1973), la corriente principal de análisis de las ciencias occidentales "hace una reducción de la realidad real a la pura substancia, relegando a la forma al ámbito exclusivo de lo ilusorio o al mundo epifenomenológico de las apariencias" (Bateson, 1973: 122).

Siguiendo a Bateson (1973), la consecuencia de lo anterior es la concreción e incorporación en la episteme occidental de una falsa separación entre mente y naturaleza, en donde la mente debe ser considerada como "inmanente en el sistema completo de relaciones organismo-ambiente" (Ingold, 2000: 16), y en la cual los humanos están necesariamente incorporados, en vez de estar confinados en nuestros cuerpos individuales como en contra de un mundo natural ubicado "allá afuera" (Ingold, 2000: 18). Para la ciencia occidental, los ecosistemas y los humanos son considerados partes disectables y separables en entidades individuales. Los primeros se reconocen como campos de materia y energía, mientras que los humanos son toda forma separada de la substancia. Una dicotomía que ha generado dos tipos de ecologías perfectamente identificables en nuestra cotidianidad: la occidental o "ecología científica", y "la ecología de la vida" (Ingold, 2000: 23), esta última planteada y vivida por los grupos indígenas de Mesoamérica, una ecología "significada y significante" (Bourdieu, 1991: 71).

Para la ciencia occidental, la ecología no es más que la adición de organismos y ambiente de tal manera que ambos poseen integridad propia independientemente de las relaciones que se establezcan. En consecuencia, los 
organismos presentan especificidad antes de entrar al ambiente y el ambientees un conjunto de condicionantes físicas que restringen a los organismos que están por incorporarse. Esta "ecología profundamente antiecológica” (Ingold, 2000: 19) establece, de principio, una separación entre organismos y ambiente, los cuales se excluyen mutuamente.

En contraste, "la ecología sintiente" que plantea Ingold (2000), representada y vivida por muchos grupos étnicos de América y el mundo, es una ecología intuitiva, basada en sensaciones y consistente de habilidades, sensitividades y orientaciones simbólicas que se han desarrollado a través de una larga experiencia histórica de interrelaciones de vida y de ambiente particular, estableciendo una "indivisible totalidad, donde el mundo toma sentido respecto a mí" (Ingold, 2000: 19).

He aquí las pautas, las tramas para la construcción de una ciencia ecológica integral y holística, emic y etic, que a la vez integra lo mejor de estas dos racionalidades $y$, por supuesto, que está basada en los seres sintientes en continua interrelación con el medio. Son éstas las "llaves de significados" que reposan en un corpus, praxisy cosmos integrado; mensajes culturales que se desprenden del mundo físico e inmaterial, que son entendidos por la educación sensorial que se practica, que residen subterráneamente en los grupos étnicos y que aún la ciencia occidental no logra detectar.

\section{El conocimiento local relativo a la milpa y la siembra del maíz. La asociación maíz-suelo}

La naturaleza transformada por la acción humana, como la realizada por los tseltales de la Sierra Norte de Chiapas entorno a su utilización como espacio productivo y de vida, contiene un conjunto de elementos materiales y simbólicos específicos que se materializan en la manera particular como los tseltales se apropian y le dan uso a estos espacios, al desarrollo de tecnologías y a la generación de conocimiento en profunda conectividad con las características y vocaciones de este contexto ecológico. En esta "arena se desarrollan los procesos sociales y las acciones simbólicas" (Barabas, 2003: 42) particulares de este grupo humano y allí se condensan sus significados de índole mítica e histórica, configurando las estructuras, las categorías y los modos de vida que los caracterizan, predominantemente asociados con la producción agrícola. A continuación se revisan algunas de estas especificidades agroproductivas con la intención de reflexionar sobre la manera particular como este grupo humano genera un conocimiento local complejo y pertinente.

La heterogeneidad ambiental y microclimática de esta región montañosa de Chiapas configura una riquísima diversidad de espacios productivos. En este contexto ecológico, "espacio vivido" (Mançano, 2008: 17), la milpa ha sido durante miles de años el eje productivo esencial de subsistencia de los grupos étnicos que han habitado la región. Dentro de este complejo y heterogéneo mosaico productivo, el maíz tiene una importancia fundamental.

Al respecto, los productores tseltales han aprendido empíricamente a identificar los parámetros subyacentes a esta diversidad de sistemas agroecológicos, nichos ecológicos y pisos altitudinales. Un rasgo de este corpus-praxis complementario consiste en la selección y preservación de las semillas o germoplasma a lo largo de todo el ciclo productivo de la milpa, una práctica que se ha extendido de manera continua desde mucho antes de la llegada de los europeos, lo que ha permitido no sólo preservar sus germoplasmas locales, sino también generar y diseñar nuevas variedades de maíz.

En la Sierra Norte de Chiapas, estas actividades agroproductivas forman parte de una secuencia histórica ininterrumpida sustentada en la utilización de una determinada praxis, generadora de un corpus de conocimiento, en el cual el pegamento metafísico de un cosmos integrador les aporta apoyo y sustento inmaterial. Se genera así una tríada: praxis, corpus y cosmos, marco existencial vital en el cual los indígenas tseltales han establecido una organización determinada del tiempo y del espacio que les permite gestionar sus entornos a partir de su propio sistema de conocimientos, y que puede describirse como "sabiduría local tseltal". 
Debido a que se está debilitando la fuerza de la naturaleza, ahora la preocupación de los tseltales es de que si se deja que se debilite nuestra madre tierra pronto ya no se va a producir maíz y para los tseltales el maíz es la base de la vida, es la que guarda la cultura, es la raíz de poder identificarse como indígena tseltal mayense, porque a través del maíz se realizan los ritos, el convivio, la armonía, el respeto el uso de la razón y la sabiduría (Grupo focal, cuidadores de la madre tierra, Bachajón, 2013).

Como era de esperar, en toda la región se cultiva maíz y los productores siembran por lo menos dos variedades: una de menor rendimiento, de maduración temprana y resistente a las heladas, y otra de mayor rendimiento pero de maduración más lenta. No obstante, muchos productores tseltales siembran más de dos variedades de maíz y en muchas comunidades es común encontrar hasta 14. Una muestra de dicha diversidad, de la frecuencia de siembra y del cultivo en la región se presenta en el cuadro l, que contiene una síntesis de investigaciones previas realizadas en la región de estudio en el marco de acompañamientos al equipo de facilitadores tseltales de las Unidades de Desarrollo Sustentable (DISO) y Medios de Sustentabilidad (MESUbYeT) del Centro de Derechos Indígenas (CEDIAC) en Chilón (ver cuadro l).

El número de variedades empleado depende del tipo de riesgos de pérdida de la cosecha que enfrentan los productores en cada ciclo, pero también se define en función del tipo de uso final - alimentario o ritual - que cada comunidad o campesino asigne al producto final de su cosecha. Desde el punto de vista de los productores tseltales, los factores más importantes que determinan la selección de semillas son el tipo de suelo, su resistencia a la sequía y el viento, su respuesta a los insumos externos, el período crítico de vulnerabilidad a las malezas, el período óptimo de fertilidad, su rendimiento, los diferentes usos del maíz —doméstico alimentario, ritual, para la venta-, la conservación poscosecha y consideraciones alimentarias - sabor, textura del grano y color-.
Por otra parte, la interacción genética ambiental intensa que exhibe el maíz "le permite adaptarse a ambientes sumamente diferentes" (Perales, 2010: 5). La temperatura media de la región de estudio, en las temporadas de siembra del maíz, puede superar los 37 grados Celsius en verano y bajar hasta los 4 grados Celsius en enero. Adicionalmente, las variedades locales pueden ser cultivadas a alturas que varían entre los 300 y los 2000 metros sobre el nivel del mar. Pueden sembrarse en suelos totalmente irrigados o en terrenos semiáridos, en planicies o en terrenos escarpados y de alta inclinación, con ciclos de crecimiento que varían normalmente entre tres y cinco meses. Es así como los productores tseltales realizan todos los años un servicio ambiental de importancia vital — que poco a poco está siendo reconocido- como conservadores de la gran variabilidad genética que ha logrado el maíz en los ecosistemas de México. No es coincidencia que los espacios naturales mejor preservados del país se encuentren habitados por grupos étnicos (Toledo y Barrera, 2008).

La habilidad para conservar, seleccionar y mejorar estos recursos genéticos depende de ciertos factores relacionados con los ámbitos familiar, social o institucional. En la esfera del hogar, los cultivadores individuales transmiten de una generación a otra la información necesaria para seleccionar las semillas que serán utilizadas en agroambientes disímiles. La transmisión exitosa de esta información sofisticada no es tarea fácil porque requiere de un proceso prolongado de entrenamiento de la vista y el tacto para identificar con destreza diferentes colores, tamaños y texturas, y obtener una selección óptima de variedades. A esto debe sumarse el talento para conocer cómo variedades distintas de semillas se adaptan a diferentes tipos de suelo y a condiciones agroambientales variables -humedad, textura, predisposición para los vientos fuertes o heladas tempranas-.

Las semillas que los productores seleccionan son ricas en mecanismos genéticos que les permiten resistir todo tipo de plagas, como las de temporal, incluidos los gusanos y los hongos, o crecer incluso cuando las primeras lluvias se interrumpen y las 
plantas se encuentran en la etapa más vulnerable de su ciclo de crecimiento. Hay muchas semillas que pueden engendrar variedades de crecimiento rápido que maduran en un lapso de tiempo muy corto, lo que constituye una característica muy importante cuando se siembra maíz en tierras altas debido al riesgo de heladas tempranas. Algunas semillas dan origen a plantas que se adaptan bien a suelos pobres, caracterizados por su alto índice de acidez o de alcalinidad; otras "vienen dotadas de un pericarpio - la capa protectora que rodea a la semilla - duro y resistente, que permite al maíz conservarse durante más tiempo, un rasgo importante bajo condiciones tropicales" (Nadal, 2000: 17).

Esta conservación in situ de los recursos genéticos es un proceso dinámico en el que los productores tseltales conservan complejos de germoplasma adquiridos con el tiempo; pero también hacen conservación en el contexto de intercambios con otros agricultores y comunidades. La experimentación con otras variedades y la obtención de nuevas son por lo tanto procesos que conducen a la utilización, conservación y refinamiento de las variedades locales debido al flujo de material genético a través de múltiples ciclos de cultivo. Ejemplo de la estrecha interacción existente entre la diversidad cultural y la variabilidad genética del maíz "es la cantidad de idiomas de pueblos indígenas que identifican un número mayor de etapas en el crecimiento y desarrollo de la planta y que expresan mayor riqueza en su descripción anatómica que lo que aparece en la literatura técnica convencional" (Nadal, 2000: 22).

El conocimiento local del que hablamos es el que resulta de la experiencia acumulada y compartida por muchos hombres y mujeres durante largo tiempo. Por ejemplo, lo que un campesino sabe acerca de la madre naturaleza es el fruto de lo que aprendió de sus padres y de sus abuelos, "pero también de lo aprendido por sí mismos, día a día, mes con mes, año con año durante su vivir, convivir y trabajar... un conocimiento detallado y profundo de la naturaleza que lo rodea" (Toledo, 2000: 10); vale decir, un proceso de indagación y experimentación tan lógicamente inducido y científico como el occidental.

\section{Los usos del suelo y las secuencias ecológicas en el conocimiento etnoecológico tseltal}

En esta investigación se encontró que, para caracterizar los tipos y la utilidad de los suelos que se encuentran en sus microrregiones, los tseltales utilizan una estructura taxonómica superior y más sofisticada a la "científica" de occidente.

$\mathrm{Al}$ respecto, el color del suelo lo utilizan como criterio para distinguir sus diferentes tipos. Existen cuatro grupos principales de suelos en la zona de estudio (ver cuadro 2).

Estos tipos de suelos son, a su vez, subdivididos o caracterizados según sus consistencias o texturas; por ejemplo, el suelo amarillo y el suelo rojo pueden ser a la vez suelos chiclosos o arenosos. Existen zonas donde sólo hay suelos pedregosos que no sirven para sembrar, o suelos secos donde siembran maíz, pero con resultados muy pobres. Basados en sus experiencias y en el conocimiento de los suelos locales, aseguran que en la tierra más fría predominan los suelos negros, en la tierra menos fría los suelos amarillos y en la tierra caliente los suelos rojos.

Al respecto, hay otro criterio muy sui generis que usan para distinguir los tipos de suelo, el cual está relacionado con el microclima del lugar. Así, un suelo puede ser también frío, sikil qu'inal, o caliente, kichin qu' inal, dependiendo de la altitud y de la cantidad de radiación que recibe del sol como consecuencia de la estación del año, lo que proporciona diferentes exposiciones solares por las sombras que se proyectan entre los cerros en las diferentes épocas. Lo anterior hace pensar que los tseltales reconocen y manejan un amplio registro de microclimas, producto de la topografía sumamente accidentada en la que habitan y de la interrelación con las vocaciones y aptitudes de cada tipo de suelo.

En ese sentido, durante los recorridos se pudieron distinguir hasta cinco tipos de relieves (ver cuadro 3).

Adicionalmente, se comprobó el reconocimiento que hacen sobre el tipo de pendiente de los terrenos. Se pudieron distinguir y caracterizar cuatro grandes grupos (ver cuadro 4). 
En el diagrama 1, transepto realizado por un equipo de trabajo durante una evaluación rural participativa en la comunidad de Sanulha', municipio de Sitalá, se recoge y sintetiza el corpus y la praxis del conocimiento previamente descrito del entorno comunitario y sus usos del suelo (ver diagrama 1).

Por último, el maíz tiene una capacidad de adaptación alta a distintos tipos de suelos, ya sean estos ácidos o alcalinos, o con variadas estructuras y texturas que determinan su contenido de nutrientes y sus propiedades de drenaje - desde suelos arenosos hasta aquellos ricos en arcillas-. Es esta capacidad asombrosa de adaptarse a los ambientes diversos que ofrece la topografía local quebrada, con su enorme variedad de nichos ecológicos que hacen del paisaje un rico mosaico ecológico, lo que convierte al maíz en el aliado perfecto para minimizar los riesgos de subsistencia, de aquí su inmenso valor para la permanencia de sus modos de vida.

Por lo que respecta a las unidades de paisaje (ver figura 1), los productores tseltales de la región realizan una caracterización principalmente a partir del reconocimiento estructural de la vegetación local, su composición florística y el uso que se da tanto a las plantas como a los árboles. En ese sentido, toda familia campesina puede utilizar una gran variedad de recursos naturales cercanos o no tan cercanos. Además de la milpa (ćalquetic), los huertos familiares (yawil ts'unub awalil) y los potreros para ganado vacuno (wacax), que representan las unidades de paisaje transformadas por el hombre, los tseltales reconocen y distinguen entre la selva alta, llamada por ellos montaña (toyem qu'inal), y las diferentes unidades vegetativas, selva secundaria o acahuales (wanc'altic), que resultan del proceso de descanso para la recuperación de la milpa o del potrero.

Respecto a la selva alta o montaña, éstos son espacios donde existe vegetación madura sin modificar y corresponden principalmente a las zonas más escarpadas o mero saulej; tierras utilizadas para recolección, caza y leña, pero que no se tumban por ser un suelo muy pedregoso no apto para la agricultura o porque se ha protegido por decisión comunal. En adición a lo anterior, las comunidades podían distinguir entre las unidades de vegetación manipuladas, por ejemplo, las selvas secundarias en reposo (acahual) o las dedicadas al cultivo del café que generan "jardines de cafetales" (Toledo et al., 2003: 7), bosques biodiversos, ricos en biodiversidad y especies, o destinados a la extracción de madera para combustible, y las selvas altas no manejadas (ver cuadro 4).

La dinámica de la sucesión ecológica está muy presente en el corpus y en la praxis en uso de ambas comunidades tseltales $y$, aunque con algunas diferencias respecto a los tiempos de descanso de los acahuales — de cuatro a cinco años en Sanulha' y de uno a dos años en San Agustín, lo cual se explica por la escasez del recurso tierra en la segunda comunidad-, se pueden identificar al menos cuatro etapas, o estadios naturales o modificados, cada uno con diferente maduración, rasgos estructurales y composición de especies, como puede comprobarse en el cuadro 4, producto de una evaluación rural participativa en la comunidad de San Agustín el Mirador, municipio de Sitalá.

\section{El cuidado de la tierra. Cuidadores de la madre tierra,jc'anan lum k'inal}

El cuidado de la tierra en la cultura tseltal es un concepto muy amplio y complejo. La tierra no es simplemente la "tierra" en su sentido físico o geográfico, sino que es "la madrecita". Esta utilización metafórica para definir las relaciones que se establecen entre el humano y su entorno establece profundos vínculos o "redes de significados" (Geertz, 1993), herencia de los antecedentes cosmológicos mayas y que de alguna manera siguen operando como ligazón cultural viva entre los tseltales.

El proceso de creación e incorporación del cargo de cuidador de la madre tierra jcanan lum k'inal es uno de los más recientes dentro del sistema de cargos que impulsa la Misión de Bachajón en el territorio tseltal. Desde 2002 comienza a gestarse la necesidad de establecerlo, inspirado en un antiguo cargo que operaba en las sociedades mayas antes de la llegada de los europeos. Al respecto, De Vos (1980) reporta: 
También veneran como señor guarda de la montaña y del pueblo al indio que hasta hoy llaman en algunas provincias Canan Lum [...] a este guardián lo llamaban también León del Pueblo, lo que hace pensar en los cuatro Balam o Jaguares que cuidaban, según los mayas, las cuatro esquinas de los pueblos y las milpas. (De Vos, 1980: 23 citando a Núñez de la Vega y a Thompson).

Pero es en 2004 cuando termina de incorporarse a la estructura de cargos tseltales, mezcla de cargos tradicionales y de más reciente creación. Las responsabilidades del cuidador y sus modos de actuación son sencillos y directos. El primer trabajo que se realiza es el siguiente: en una visita a una comunidad se ubica a un posible candidato que reúna un conjunto de aptitudes y el sistema de cargos local debe proponerlo y aprobarlo. Una vez que el candidato ha aceptado y asistido a las primeras charlas, donde se estudian temas de sensibilización religiosa y agroecología productiva, junto con el equipo promotor de la Misión pasa a evaluar las condiciones locales de su comunidad.

Ya en esta primera visita se obtiene un panorama de la situación que ésta vive y, a partir de ello, priorizan los problemas relacionados con la agricultura y la soberanía alimentaria. A partir de lo anterior, se establece un programa de motivación y animación según el cuadro de necesidades que presente la comunidad. Idealmente, ya el ćanan en esta etapa se ha incorporado a la estructura, una "toma de cargo" que se realiza anualmente en encuentros comunitarios de cargos llamados tsumbalil. En lo sucesivo recibirá formación y proseguirá sus capacitaciones para desarrollar iniciativas de producción agroecológicas en su comunidad. Ésta es la manera como el conocimiento y la diversificación de prácticas productivas se ha ido derramando paulatinamente y de campesino a campesino hacia los miembros de cada comunidad en el territorio.

Pero dejemos que sean los mismos cuidadores los que nos recreen ese mundo de simbolismos o "llaves de entendimiento" mediante algunas narrativas que expusieron en una dinámica grupal realizada en uno de los tsumbalil:
Es el entendimiento y el trato que le damos a la naturaleza en el trabajo y en nuestra vida que es de forma equilibrada y armónica. El cuidado es un concepto que va después, antes de esto va el concepto de la tierra. La tierra para los tseltales la ven, la aman, la quieren, la respetan y la veneran porque según el pensamiento indígena que ha sido transmitido de generación en generación es de que la tierra es sagrada y es como nuestra madre debido que de ahí nos sustentamos.

La dedicación que se le da a la tierra más que nada en el trabajo del campo, así como los ritos que se hace antes de tumbar el monte para la siembra, el rito de la santa cruz y la ofrenda a la sagrada tierra y de tener en lo profundo de la mente, en el corazón, y más allá de la conciencia, el amor a la tierra y el gran significado del maíz.

Los antepasados cuidaban mucho a la tierra y se podría decir que lo cuidaron de dos formas. Cuidado de manera agroecológica mediante la rotación de cultivo, asociación de cultivo, siembra de plantas de cobertura, plantas aromáticas como repelente de plagas y entre otras plantas comestibles con la función de cumplir la riqueza de la cadena alimenticia que la propia naturaleza va formando. Cuidado de manera religiosa y espiritual: por la tierra tenían gran respeto y sentían mucho amor. El significado de la tierra para ellos es mucho más que la madre, por eso la veneran, le dan ofrenda, en sí se entregan con ella y sienten ser como esclavos de la tierra.

La tierra da todo como una madre, pero igual se agota si no hay una buena alimentación, si no hay amor, escucha y comprensión. Para los tseltales, la tierra es en sí la naturaleza que en ella forman los árboles, los animales, el agua, el suelo, el aire, etcétera. Para los árboles pedir permiso con Dios para tumbarla y tumbar cuando sea necesaria. Igual con los animales, porque psicológicamente los campesinos dicen que el árbol tiene vida y que responde cuando hay desequilibrio; igual con los animales, dicen que, si la matamos nada más por gusto, que responden los guardias de los cerros. La sagrada tierra es nuestro sustento, por eso es 
indispensable y de vital importancia el cuidado de ella, porque, depende del cuidado que le damos en el trabajo y en la vida, es la calidad y cantidad de alimentos que se nos proporciona. (Grupo focal, cuidadores de la madre tierra, Bachajón, 2013).

Estas prácticas de "ecología sagrada” (Berkes y Folkes, 2000) que establecen en el universo natural estructuran un conjunto de creencias y ceremonias rituales; una cosmovisión politeísta y natural que persiste semioculta y amestizada en toda América, "rituales agrícolas, sitios sagrados, calendarios de ceremonias religiosas y toda una gama de deidades ligadas con los elementos de la naturaleza, son algunos indicadores del sincretismo encontrado en estos pueblos" (Toledo et al., 2003: 83).

\section{Conclusiones}

Con frecuencia se ha considerado la modernización del agro y sus prácticas asociadas a la producción científica y moderna como la palanca indispensable para dejar atrás la supuesta irracionalidad y el atraso de los modos de producción campesinos y étnicos, así como el corpus y la praxis de conocimiento que éstos despliegan al tratar de hacer productivos y sustentables sus modos de vida. El meollo del asunto es que "tal modelo ha sido hecho verdad" (Ploeg, 2010: 40) y, a pesar de que este "aparato ideológico y hegemónico del desarrollo” (Escobar, 1995: 43) se ha desnudado en su verdadera dimensión en los últimos 60 años, el paradigma de la modernización sigue persistiendo como "modelo en las políticas agrarias y en las ciencias aplicadas" (Ploeg, 2010: 40), en detrimento de las lógicas de producción y "epistemologías del sur" (De Souza, 2009).

En esta investigación se demuestra cómo los indígenas tseltales de la Sierra Norte de Chiapas implementan una triada unificada de cosmos, corpus y praxis en sus estrategias productivas, la cual se ha desarrollado en el tiempo a partir de una apropiación particular, empírica y pertinente de interrelaciones complejas hombre-ambiente, cuyas "dinámicas se traducen en acciones concretas que los hacen actores sociales vitales" (Hernández et al., 2011: 132) de su propio desarrollo autógeno.

Al respecto, esta investigación reporta cómo los productores tseltales de microparcelas de la Sierra Norte de Chiapas conocen y utilizan un conjunto de categorías de conocimiento agroproductivoedafológico-topográfico, y poseen un conocimiento amplio y profundo de las condiciones ecológicas de sus entornos, todo lo cual, asociado al conocimiento de las características fenotípicas de las distintas variedades de maíz que manejan, les permite seleccionar la más adecuada para cada microrregión. Debe señalarse que esta selección no puede considerarse, bajo ningún aspecto, como un método al tanteo de prueba y error, sin coherencia ni finalidad, sino que se trata de un proceso claramente orientado, por lógica y racionalidad empírica, a un fin que se concreta dentro del marco global de episteme particular generador de conocimiento local. A través del proceso señalado de observación, interpretación, valoración y manipulación, se amplía el ámbito del conocimiento y se hace una adaptación muy afinada de las variedades locales de maíz, lo cual les permite adquirir nuevas percepciones y acrecentar su acervo cultural.

En otro orden de ideas, se puede concluir que el campesino tseltal posee un amplio conocimiento ecológico sobre su entorno y una dinámica sucesional a medida que introduce cambios en el uso del suelo, pues no sólo reconoce en sus paisajes las formas del terreno, la inclinación o pendiente, el tipo de suelo y la clase de vegetación afectadas, sino que también puede tomar decisiones "sabias" respecto a los tiempos y los modos de usarse un espacio determinado según su vocación y capacidades. Este conocimiento les sirve para planear el uso de sus recursos y decidir qué hacer con cada microrregión a partir de sus aptitudes, vulnerabilidades y riesgos durante todo el año.

También deben mencionarse las estrategias o mecanismos de manejo que utilizan para buscar reducir los impactos de uso sobre los ecosistemas, los cuales mantienen e incrementan su estabilidad y resiliencia. Entre ellos, resaltar el mantenimiento de sus entornos naturales y manipulados, constituidos por una alta 
diversidad de paisajes, especies y variedades, que establecen mosaicos espaciales productivos con usos de suelo distintos, con policultivos y germoplasmas locales, y en donde se establecen estrategias de rotación tanto espacial como temporal, "lo cual resulta en una manipulación integrada de procesos a diferentes escalas de tiempo y espacio" (Toledo et al., 2003: 115).

A esta habilidad autorreguladora y flexible para modificar las estrategias de manejo de acuerdo a los efectos producidos sobre los recursos que utilizan se la conoce como "manejo adaptativo" (Berkes el al., 2000), siendo éste uno de los paradigmas fundamentales sobre los cuales se sustentan sus esquemas productivos de subsistencia.

Como se ha evidenciado a lo largo de este ensayo, el conocimiento local, a pesar de su riqueza y complejidad, parecería que no ha logrado integrarse al esquema científico, ontológica y epistémicamente estructurado por los cánones occidentales. De alguna manera, sus prácticas y sus métodos sui generis caen fuera del ámbito del esquema científico aceptado por la ciencia occidental; en consecuencia, los campesinos y productores indígenas, en cuanto sujetos activos y entendidos, capaces de mejorar sus propias condiciones, son marginados del ámbito del desarrollo rural.

Al respecto, la investigación es concluyente respecto a la necesidad de que las nuevas propuestas y políticas de desarrollo eliminen las visiones y presupuestos etnocéntricos que se originan desde el núcleo diseñador de políticas y entiendan las complejas interrelaciones de los fenómenos sociales, económicos y ambientales de los grupos étnicos de Chiapas y Mesoamérica. Así pues, los nuevos enfoques de desarrollo deben considerar el conocimiento local e indígena. Tratar de establecer vínculos con las instituciones locales es un primer paso, e implementar estrategias diagnósticas basadas en la triada praxis, corpus y cosmos se constituye en un requisito indispensable a fin de acercarse a cada particularidad local. Se trata de un reto que conlleva comprender y demoler los viejos paradigmas cientificistas, una pesada inercia que todavía revolotea fuertemente en nuestros gobernantes y sus diseñadores de políticas.

\section{Referencias bibliográficas}

Barabas, Alicia (coord.) (2003), Diálogos con el territorio, t. II, México: INAH/CONACULTA.

Bateson, Gregory (1973), Steps to an Ecology of Mind, Nueva York: Paladin Books.

Berkes, F., J. Holding y C. Folke (2000), "Rediscovery of traditional ecological knowledge as adaptative management", en Ecological Applications, núm. 10, pp. 1251-1262.

Bourdieu, Pierre (1991), El sentido práctico, Madrid: Taurus. Broda, Johana (1991), "Cosmovisión y observación de la naturaleza: el ejemplo de los cultos de los cerros en Mesoamérica", en Johana Broda, S. Iwaniszewski y Lucrecia Maupomé (eds.), Arqueoastronomía y etnoastronomía en Mesoamérica, México: UNAM.

Broda, Johana y Félix Báez (2001), Cosmovisión ritual e identidad de los pueblos indígenas de México, México: FCE/ CONACULTA.

Broda, Johana, S. Iwaniszewski y Arturo Montero (2007), La montaña en el paisaje ritual, México: IIHUNAM/INAH.

Bonfil, Guillermo (comp.) (1993), Identidad, cultura $y$ modernidad. Hacia nuevos modelos de relaciones interculturales, México: CONACULTA.

Cardoso de Oliveira, Roberto (2007), Estructura social $y$ etnicidad, México: CIESAS/UAM/Universidad Iberoamericana.

De Souza, Boaventura (2009), Epistemologías del sur, México: CLACSO/Siglo XXI.

De Vos, Jan (1980), La paz de Dios y del rey. La conquista de la selva Lacandona por los españoles, 1525-1821, Chiapas: Gobierno del Estado.

Escobar, Arturo (1995), Encountering Development. The Making and Unmaking of the Third World, Nueva Jersey: Princeton University Press.

Gadgil, M. y R. Guha (1992), This Fissured Land: An Ecological History of India, California: University of California Press.

Gandarilla Salgado, José Guadalupe (2011), "Reseña de Una epistemología del Sur. La reinvención del conocimiento y la emancipación social", en Coloquio Internacional hacia la Construcción de un Nuevo Paradigma Social, 
México: UAM-Xochimilco.htttp://csh.xoc.uam.mx/ produccioneconomica/Coloquio_nuevoparadigma/ archivos Nuevo Paradigma/Boaventura Rese\%C3\%Bla.pdf> [29 de mayo de 2013].

Gardner, Katy y David Lewis (2003), Antropología, desarrollo y el desafío posmoderno, México: El Colegio Mexiquense.

Geertz, Clifford (1993), Descripción densa: hacia una teoría interpretativa de la cultura, España: McGraw-Hill.

González Jácome, Alba (1993), "Management of Land, Water and Vegetation in Traditional Agroecosystems in Central México", en Landscape and Urban Planning, núm. 27, pp. 141-150.

Giménez, Gilberto (1999), "Territorio, cultura e identidades", en Estudios sobre las Culturas Contemporáneas, época II, vol. V, núm. 9.

Guevara-Hernández, Francisco, Max McCune Nils, Luis Rodríguez-Larramendi y Gillian Newell (2011), "Who's Who? Power Mapping, Decision Making and Development Concerns in an Indigenous Community of Oaxaca, Mexico", en Human Ecology, vol. 36, núm. 2, pp. 131-144.

Guevara-Hernández, Francisco, Nils McCune, José Nahed-Toral, Paula Mendoza-Nazar, Jesús OvandoCruz, Benigno Ruiz-Sesma y Leopoldo MedinaSanson (2012), "Social-Ecological Resilience and Maize Farmingin Chiapas, Mexico",en SimeCurkovic (ed.), Sustainable Development: Authoritative and Leading Edge Content for Environmental Management, s.l.: Intech Open Sciencie. 〈http://www.intechopen.com/books/ sustainable-development-authoritative-and-leadingedge-content-for-environmental-management/ social-ecological-resilience-and-maize-farming-inchiapas-mexico> [26 de mayo de 2013].

Ingold, Tim (2000), The perception of the Environment. Essays on livelihood, dwelling and skills, Reino Unido: Routledge.

Mançano Fernandes, Fernando (2008), "Sobrela tipología de los territorios", s.l.: s.e. 〈http://ebookbrowse. com/bernardo-tipologia-de-territorios-espanolpdf-dl49176315> [26 de mayo de 2013].

Nadal, Alejandro (2000), Biodiversidad, sustento y culturas. El caso del maíz mexicano en el NAFTA: variabilidad genética y liberalización comercial, México: Programa de Ciencia y Tecnología-El Colegio de México.

Perales Rivera, Hugo (2010), Paisajes de maíz en México: evolución y conservación de recursos genéticos in situ, San Cristóbal de Las Casas: El Colegio de la Frontera Sur.

Ploeg, Jan Douwer van der (1990), "Sistemas de conocimiento, metáfora e interfaz: el caso del cultivo de la patata en el altiplano peruano", en Agricultura y Sociedad, núm. 56, pp. 143-166.

Ploeg, Jan Douwer van der (2010), Nuevos campesinos. Campesinos e imperios alimentarios, España: Icaria.

Richards, P. (1980), "Community environmental knowledge in African rural development", en D. W. Brokensha, D. M. Warren y O. Werner (eds.), Indigenous Knowledge Systems and Development, Lanham, Maryland: University Press of America, pp. 183-203.

Richards, P. (1993), "Cultivation: knowledge or performance?", en M. Hobart (ed.), An Anthropological Critique of Development: the Growth of Ignorance, Londres: Routledge, pp. 61-78.

Rodríguez Moreno, José (2006), Etnoecología y desarrollo sustentable en dos comunidades indígenas de los municipios Chilón y Sitalá, Chiapas, (tesis inédita de maestría en medioambiente y desarrollo), Universidad Iberoamericana de Puebla, Puebla.

Toledo, Víctor (2000), Ecología, espiritualidad y conocimiento, México: Universidad Iberoamericana de Puebla/PNUMA.

Toledo, Victor, B. Ortiz-Espejel, P. Moguel y M. Ordóñez (2003), "The multiple use of tropical forest by indigenous people in México: a case of adaptative management", en Ecology and Society, vol. 7, núm. 3. 〈http://www.ecologyandsociety.org/vol7/iss3/art9/> [23 de mayo de 2013].

Toledo, Víctor y Narciso Barrera-Bassols (2008), La memoria biocultural. La importancia ecológica de las sabidurías tradicionales, Barcelona: Icaria.

Warman, Arturo (1988), La historia de un bastardo, maíz y capitalismo, México: UNAM-Instituto de Investigaciones Sociales/Fondo de Cultura Económica. 
Cuadro 1. Tabla de correspondencia tseltal-español de las variedades locales y porcentaje reportado de la variedad

\begin{tabular}{|c|c|c|}
\hline $\begin{array}{c}\text { Variedad de maíz según su nombre } \\
\text { en tseltal }\end{array}$ & $\begin{array}{l}\text { Variedad de maíz según traducción } \\
\text { directa del tseltal al español }\end{array}$ & $\begin{array}{c}\text { Porcentaje reportado de la } \\
\text { variedad }\end{array}$ \\
\hline c’anal & amarillo & $34 \%$ \\
\hline saquil & blanco & $25 \%$ \\
\hline saquil pequ’el & blanco pálido & $1 \%$ \\
\hline ijc ${ }^{\prime} a l$ & negro & $18 \%$ \\
\hline ijc`alpat & negro en la superficie & $1 \%$ \\
\hline ijc 'alte' & negro claro & $1 \%$ \\
\hline sac waj & blanco de elote chico (liviano) & $6 \%$ \\
\hline wujul & pinto (amarillo-morado-blanco) & $2 \%$ \\
\hline wujul ijc`al & pinto oscuro & $1 \%$ \\
\hline tsaj & colorado & $2 \%$ \\
\hline yax & morado claro & $1 \%$ \\
\hline yaxte & morado oscuro & $3 \%$ \\
\hline chaparro & chaparro & $1 \%$ \\
\hline wujultic & pinto morado-blanco & $1 \%$ \\
\hline sejk’ej`ub & arcoíris, con al menos 12 colores & $1 \%$ \\
\hline
\end{tabular}

Fuente: CEDIAC (Centro de Defensa del Indígena, de Chilón, Misión Jesuita de Bachajón), 2007-2012

\section{Cuadro 2. Tipos de suelos según taxonomía tseltal}

\begin{tabular}{|c|c||}
\hline Tipo de suelo según su nombre en tseltal & Tipo de suelo según traducción del tseltal al español \\
\hline tontikil & suelo negro \\
\hline \hline chabeclum & suelo amarillo \\
\hline \hline tzajalum & suelo rojo \\
\hline tzajalguecz & suelo café-rojizo \\
\hline \hline
\end{tabular}

\section{Cuadro 3. Tipos de relieves}

\begin{tabular}{|c|c|}
\hline Tipo de relieve según su nombre en tseltal & Tipo de suelo según traducción del tseltal al español \\
\hline \hline sawlej & ladera \\
\hline bawitz & meseta \\
\hline slomlej & planada \\
\hline \hline lomerío & lomerío \\
\hline \hline yokwits & pie de monte \\
\hline
\end{tabular}


Cuadro 4. Taxonomía topográfica utilizada por los tseltales para caracterizar los tipos de suelos según su pendiente

\begin{tabular}{|c||c||}
\hline Tipo de terreno según su pendiente, en tseltal & Tipo según traducción del tseltal al español \\
\hline \hline spamlej & terrenos de poca pendiente de $0^{\circ}$ hasta los $7^{\circ}$ \\
\hline \hline medio spamlej & terrenos de ligera pendiente de $7^{\circ}$ hasta los $15^{\circ}$ \\
\hline \hline saulej & terrenos con pendiente de $15^{\circ}$ hasta los $30^{\circ}$ \\
\hline \hline mero saulej & terrenos con pendiente fuerte de más de $30^{\circ}$ \\
\hline
\end{tabular}

Diagrama 1. Síntesis del conocimiento edafológico y topográfico del entorno en una comunidad tseltal

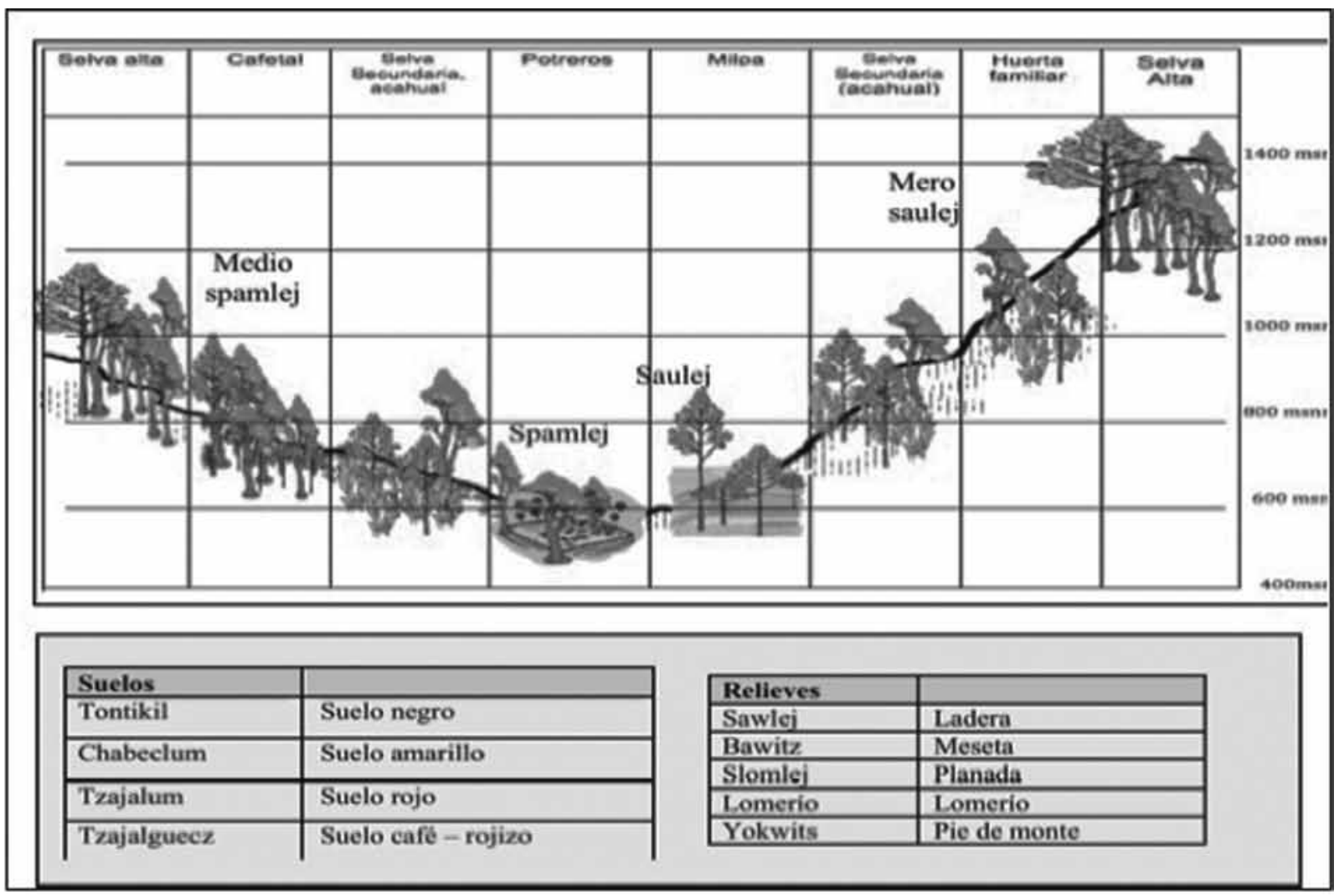

Fuente: Evaluación Rural Participativa, 2010, comunidad de Sanulhá. 


\section{Figura 1. La sucesión ecológica y cambio de usos de suelo en una comunidad sitalteca}

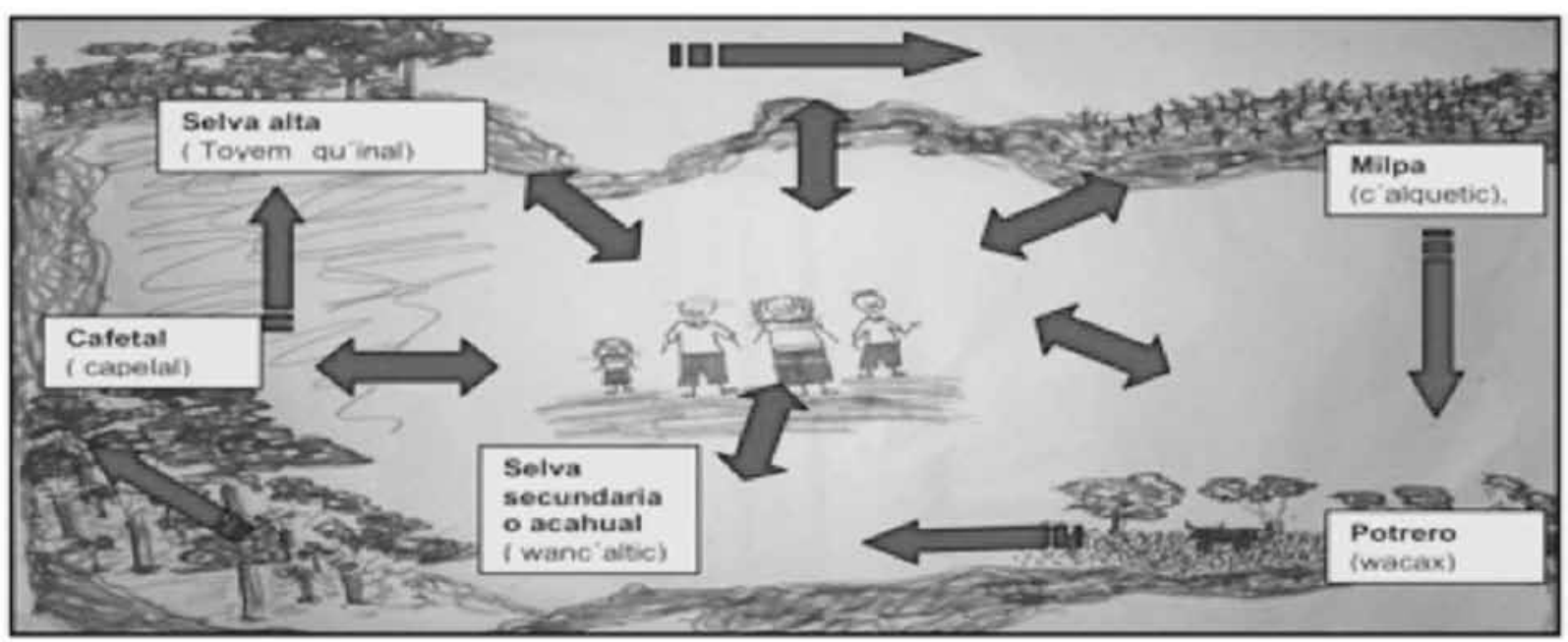

Fuente. Evaluación Rural Participativa en San Agustín el Mirador, Chiapas 2010. 
Mapa 1. Región de estudio. Municipios de Chilón y Sitalá en la Sierra Norte de Chiapas

\section{Cniapas}

Division municipal

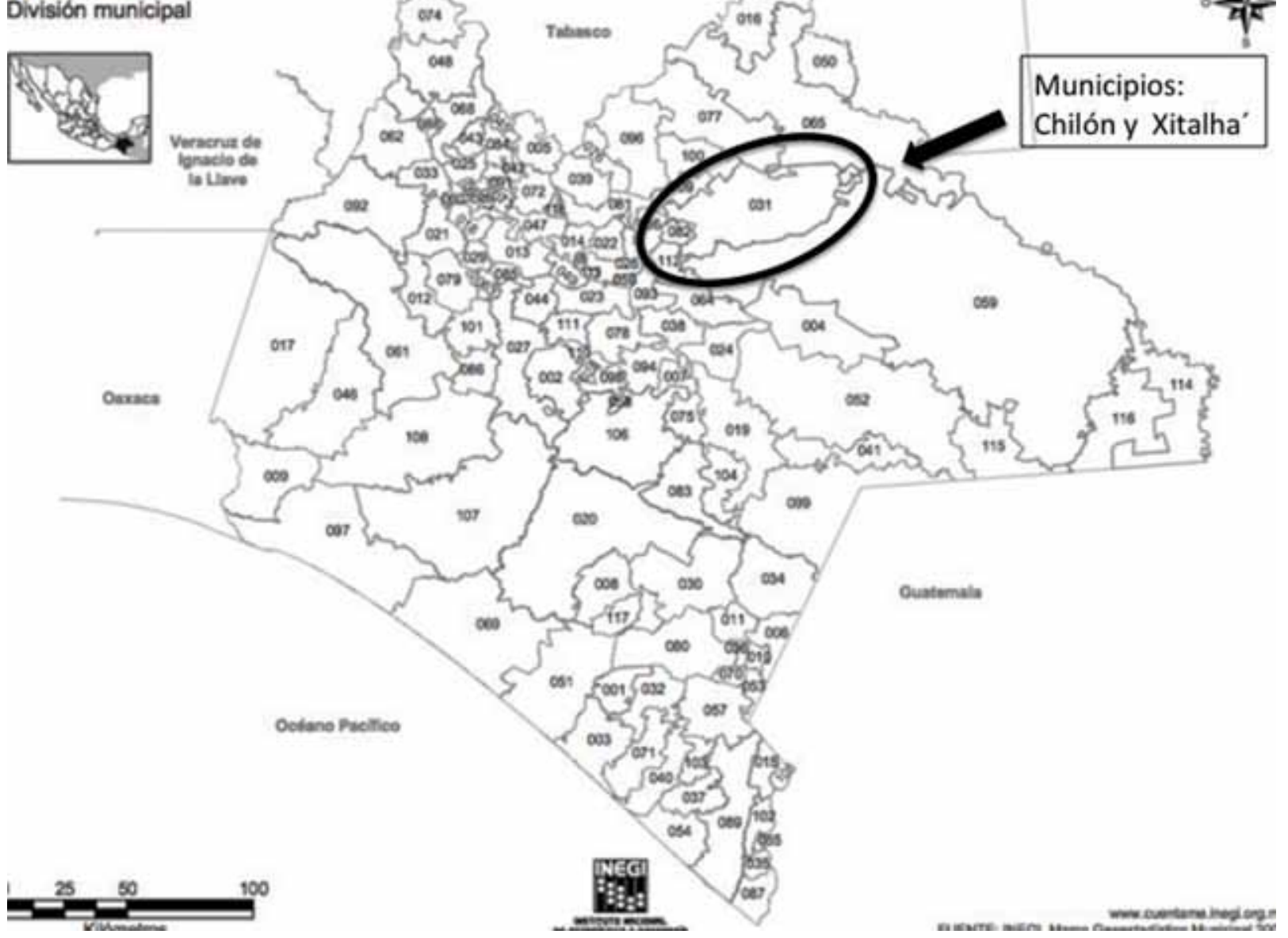

Fuente: INEGI. www.cuentame.inegi.org.mx [5 de junio de 2013]. 